\title{
Seizures and epilepsy in cats
}

This article was published in the following Dove Press journal:

Veterinary Medicine: Research and Reports

30 July 2014

Number of times this article has been viewed

\section{Sarah A Moore \\ Department of Veterinary Clinical Sciences, The Ohio State University, Columbus, $\mathrm{OH}, \mathrm{USA}$}

\begin{abstract}
Seizures are a common presenting complaint in cats, although causes and options for the treatment of seizures in this species have been historically poorly described in the veterinary literature. Seizure manifestation in cats may be different than what is typically seen in dogs, but the underlying causes of seizure activity are the same. These include primary epilepsies, structural epilepsies, and reactive seizures. Although primary epilepsy was once believed to be rare in cats, we now commonly appreciate this syndrome, albeit at a lower frequency than in dogs. Because of this, a complete diagnostic work-up is recommended for all cats presenting for initial evaluation of seizures. Symptomatic treatment of seizures in cats is similar to dogs, with only a few limitations related to species-specific antiepileptic drug toxicities. The goal of this review is to summarize the recent veterinary literature related to feline seizures, with a focus on seizure classification, clinical manifestation, diagnostic evaluation, and treatment options.
\end{abstract}

Keywords: antiepileptic drug, seizure classification, levetiracetam, zonisamide, phenobarbital

\section{Introduction}

Seizures are one of the most common neurologic disorders of cats, affecting $1 \%-2 \%$ of the general feline population. ${ }^{1}$ Causes of seizures and treatment options for cats are historically poorly described in the veterinary literature; however, there is a wealth of recently published clinical research focusing on feline seizures. ${ }^{2,3,4,5,6,7}$ Underlying causes of seizures in cats are similar to those identified in dogs, although incidence varies between the two species, as do clinical manifestations of seizure activity. The goals of this article are to review recent veterinary literature related to feline seizures, with a focus on seizure classification, clinical manifestations, diagnostics, and treatment options.

\section{Classification and seizure etiology}

A seizure is a physical manifestation of abnormal hypersynchronous electrical discharges in the brain. ${ }^{8}$ Epilepsy is a disorder of repeated seizures occurring more than 24 hours apart without an identifiable extracranial cause. Clinical classification of seizures in veterinary medicine is adapted from human schematics and can be divided into three broad categories: primary (genetic) epilepsies, secondary (structural) epilepsies, and reactive seizures. ${ }^{9}$ Signalment, neurologic examination findings, and diagnostic testing results will dictate how a patient is classified.

The term "primary/genetic epilepsy" is synonymous with the older term, "idiopathic epilepsy", and implies a documented or presumed genetic basis for seizure activity. ${ }^{8,9}$ 
As with dogs, cats with primary epilepsy tend to present at a younger age than cats with acquired structural causes of seizure activity, with their first seizure generally occurring between 1 and 7 years of age. ${ }^{2,3}$ A diagnosis of primary epilepsy implies there is no macroscopic structural abnormality of the brain to explain seizures, such as a tumor, stroke, or congenital anomaly. It also implies that metabolic cause has been excluded after thorough diagnostic evaluation. Ultimately, a definitive diagnosis of primary epilepsy can be made only through exclusion of other causes of seizure activity and by demonstration of a genetic marker for a previously described heritable epilepsy syndrome. The ability to demonstrate a genetic marker of primary epilepsy in veterinary patients is often limited, as testing for mutations associated with primary epilepsy is only available for a few canine breeds. As such, a diagnosis of presumptive primary epilepsy is often made in veterinary patients with normal diagnostic evaluations and normal neurologic examinations and who present between 1-7 years of age with their first seizure. In patients with primary epilepsy, seizures are related to intrinsic hyperexcitability of cortical neurons caused by defects in membrane ion channels or dysfunction or imbalance of neurotransmitters. ${ }^{9}$ An example of a well-described genetic epilepsy in veterinary medicine is the $L G I 2$ nonsense mutation in Lagotto Romagnolo dogs, which leads to a remitting form of juvenile epilepsy and is inherited in an autosomal recessive fashion. ${ }^{10}$

Historically, the existence of primary epilepsy in cats has been controversial among veterinary neurologists, with some authors suggesting such a syndrome is rare or nonexistent. ${ }^{11,12}$ In dogs, there are many breed-associated primary epilepsy syndromes for which the genetic basis has been described. ${ }^{13}$ Although this is not yet the case for cats, several recent largescale retrospective studies describe a population of cats who meet the clinical criteria for a diagnosis of primary epilepsy. This includes an onset of seizures between 1 and 7 years of age, a normal neurologic examination between seizures, normal magnetic resonance imaging (MRI), and normal cerebrospinal fluid (CSF) examination findings. It is important to point out that the incidence of primary epilepsy in cats presented for seizure activity is lower than what is observed in dogs, with only about 1 in 4 cats meeting the criteria for this diagnosis. ${ }^{1}$ Accordingly, structural and metabolic causes of seizures occur more commonly. Clinicians should be aware that although primary epilepsy is an important differential in cats with seizures, this diagnosis should only be made after metabolic screening, advanced imaging, and CSF analysis have excluded other underlying causes.
"Structural" epilepsy implies a macroscopic structural problem of the forebrain leading to seizure activity. Patients with congenital anomalies, brain neoplasms, infectious or autoimmune meningoencephalitis, and vascular events fall into this category. In the veterinary literature, this is also commonly referred to as "symptomatic" or "secondary" epilepsy. Commonly reported causes of structural epilepsy in cats include infectious etiologies such as feline infectious peritonitis (FIP) or toxoplasmosis, noninfectious inflammatory disease (with neoplasms with meningioma and lymphoma being the most common), and ischemic or hemorrhagic infarcts. ${ }^{1-16}$

"Reactive seizures" are seizures occurring secondary to a systemic, toxic, or metabolic abnormality in the absence of an identifiable intracranial disease process. Because these seizures do not result from an intracranial cause, they are not classified as epilepsy even if they occur repeatedly. In cats, diseases reported to cause reactive seizures include hepatic encephalopathy, severe uremia of end-stage renal disease, severe hypoglycemia, severe hyperthyroidism, severe systemic hypertension, polycythemia vera, hypocalcaemia, hypertriglyceridemia, and organophosphate exposure. Of these causes, hepatic encephalopathy, hypoglycemia, and organophosphate exposure were the most common in a recent retrospective study. ${ }^{1,17,18}$

\section{Clinical manifestations of seizures in cats}

Seizures in cats, regardless of cause, have clinical manifestations unique to the species. Although generalized tonic or tonic-clonic seizures may occur, cats are more likely to manifest focal seizures compared with dogs. ${ }^{15} \mathrm{~A}$ focal seizure is defined as a seizure in which the first clinical and electroencephalographic abnormalities indicate activation of focal portion of one cerebral hemisphere. ${ }^{19}$ Focal seizures often have concurrent somatosensory manifestations such as biting, licking, or obsessive running; oroalimentary manifestations such as excessive swallowing; or dyscognitive manifestations such as sudden behavioral arrest or altered mentation.

Of particular note is a syndrome of orofacial seizures with dyscognitive manifestation described in a cohort of European domestic shorthair cats. ${ }^{5,6}$ The cats described in these case series presented with seizures involving hypersalivation, facial twitching, lip smacking, excessive swallowing, and pupillary dilation. ${ }^{5}$ Many cats had altered mentation during these seizures, evidenced as "staring off into space". Some progressed to generalized seizures, whereas others did not. ${ }^{5,6}$ All cats that underwent advanced imaging 
were diagnosed with bilateral hippocampal pathology on MRI, which was histopathologically confirmed to represent degeneration, necrosis, and neuronal cell loss within the pyramidal cell layer or the hippocampus. ${ }^{5}$ Because of consistent involvement of the hippocampus, Pakozdy et $\mathrm{al}^{5}$ hypothesize this syndrome represents a form of limbic epilepsy. The significance of the hippocampal necrosis in these cats remains unclear and could represent primary pathology or may be the result of prolonged seizure activity. ${ }^{5,6}$ Recently, a similar familial primary epilepsy syndrome was reported in a colony of laboratory cats with normal MRI and identical ictal pattern. ${ }^{20}$ Electroencephalographic findings in this family of cats were also consistent with limbic epilepsy.

\section{Diagnostic recommendations for the feline patient with seizures}

As previously noted, the incidence of primary epilepsy in cats is lower than that seen in the canine population. As such, it is this author's opinion that all cats with repetitive seizures should have a clinical and diagnostic evaluation to exclude metabolic and structural causes. This begins with a thorough neurologic examination. The neuroanatomic localization for any patient with seizures is the forebrain, as no other region of the nervous system will produce seizure activity. ${ }^{21,22}$ The goal of the neurologic examination is to identify or exclude the presence of lateralizing forebrain signs such as unilateral decrease in menace response despite a normal pupillary light reflex, unilateral decreased response to nasal sensation, and unilateral deficits in proprioceptive placing and hopping. ${ }^{23}$ In addition, animals with lateralizing forebrain signs may circle toward the affected side, and owners may report changes in personality or learned behavior at home. ${ }^{9,23}$ Any of the aforementioned findings would warrant heightened concern for structural epilepsy, but lack of these findings does not exclude an ultimate diagnosis of structural epilepsy. Some lesions may involve relatively silent or difficult-to-assess regions of the brain, such as the olfactory lobe, and may not produce clinical signs until disease is advanced. Behavioral changes related to forebrain disease are more subtle in cats than in dogs (ie, increased hiding behavior) and may require careful questioning of the owner to identify. Clinicians should be aware that patients who have recently had a seizure may display deficits that are benign postictal changes. If present, these deficits will be symmetrical and generally resolve within a few days. ${ }^{9}$ If symmetrical neurologic deficits are observed in a patient who has recently had a seizure, the neurologic examination should be repeated in a few days to be sure other unilateral forebrain signs are not being masked by these postictal changes.

Inherent to the feline neurological examination are several challenges such that occasionally a complete examination cannot be performed. This may occur because of the fractious nature of the cat, refusal of the cat to ambulate in hospital for a gait examination, or because scruffing fractious cats for examination can produce a "clipnosis" affect that may alter the neurologic examination. ${ }^{24}$ Some feline patients are unwilling to have their feet manipulated for postural reaction testing or spinal reflexes. In these instances, tactile and visual placing tests may replace other methods of assessing proprioception. For very fractious feline patients, examination may consist solely of a gait examination and thorough history from the owner. However, it is the author's experience that most cats will be agreeable to a complete neurologic examination if the clinician proceeds slowly and completes the least offensive tests first (ie, gait examination and cranial nerve exam) before progressing to tests that involve manipulating the feet.

Metabolic screening is indicated for all cats with newonset seizures. At the author's institution, this routinely consists of complete blood count (CBC), chemistry profile including T4, and bile acids if clinical picture or chemistry profile warrants. Systolic blood pressure measurement is obtained regardless of whether cardiac or renal abnormalities are identified. At the author's institution, we find that the most reliable measurements are obtained when the cat is left alone with the owner in a quiet room to acclimate for some time before obtaining a reading, which is ideally taken before extensive manipulation for neurologic examination, drawing blood, and so on. Common metabolic causes of seizures in cats are detailed in the Classification and seizure etiology section. The goal of metabolic evaluation is to confirm or exclude these diagnoses.

For feline patients with normal metabolic screening results, advanced imaging of the brain is indicated to exclude structural causes of epilepsy. Common imaging modalities include computed tomography (CT) or MRI. MRI is preferred, as it provides superior detail of intracranial soft tissue structures. If advanced imaging is normal, CSF analysis should be performed concurrently to evaluate for infectious or immune-mediated causes of seizures. Alterations in CSF cell count and protein concentration are sensitive but nonspecific findings in a patient with seizures, and polymerase chain reaction, titers, or antigen testing for infectious organisms such as Cryptococcus, feline corona virus/feline infectious peritonitis, and toxoplasmosis may be indicated if CSF results are abnormal. 


\section{Symptomatic therapy}

Antiepileptic drugs (AEDs) available for cats are similar to those available for dogs. There are, however, a few key differences in toxicity and metabolism that are unique to cats, and of which clinicians should be aware. Table 1 details common maintenance AEDs used in cats, along with their recommended starting doses and documented adverse effects.

Phenobarbital (PB) was the first commercially manufactured AED and is the most extensively used drug in veterinary medicine. ${ }^{25}$ It is available in oral tablets, oral solution, and injectable forms and is one of the first-line AEDs for seizure control in cats. ${ }^{11,25} \mathrm{~PB}$ is primarily metabolized by the liver and induces the cytochrome P450 (CYP450) pathway, which can lead to a progressive shortening of the elimination halflife with chronic administration. ${ }^{26}$ The elimination half-life of PB in cats is approximately 40 hours, although significant variability may be noted between cats. ${ }^{27}$ The recommended starting dose in cats is $2-3 \mathrm{mg} / \mathrm{kg}$ orally twice daily, and the drug requires 10-15 days to reach steady-state concentration after initiating therapy or changing dose. ${ }^{22,27}$ The therapeutic range for $\mathrm{PB}$ in cats is generally considered to be $20-30 \mu \mathrm{g} /$ $\mathrm{mL}$ and is extrapolated from what is effective, but not toxic, in dogs. Serum PB concentrations should be measured 2-3 weeks after starting treatment and after any change in dose. Timing of blood draw does not significantly alter measured steady-state PB serum level in dogs, and the same would be predicted in cats. ${ }^{28}$ Although there are limited available efficacy data regarding PB in cats, the efficacy of PB in epileptic dogs is reported to be between $60 \%-80 \% .^{29}$ The most common adverse effects of PB reported in dogs are sedation, weight gain, increased thirst, increased urination, and increased appetite, most of which resolve within a few weeks of starting treatment. ${ }^{30}$ In the author's experience, cats are less likely to develop polyuria and polydipsia when receiving this medication. Less common but more serious adverse effects of PB administration include acute idiosyncratic liver or bone marrow toxicity occurring within 1-4 weeks of starting treatment, or acquired myelo- or hepatic fibrosis after long-term administration. Both acute and chronic toxicities may be reversible if caught early and PB is discontinued. Reports of PB-associated hepatotoxicity in veterinary medicine are exclusively limited to dogs; however, vigilance for these adverse effects in cats is warranted, and routine monitoring of serum chemistry profiles is recommended in feline patients receiving PB. Additional rare adverse effects of PB specifically reported in cats include facial pruritus, limb edema, thrombocytopenia, and cutaneous and mucosal hypersensitivity reactions, all of which were

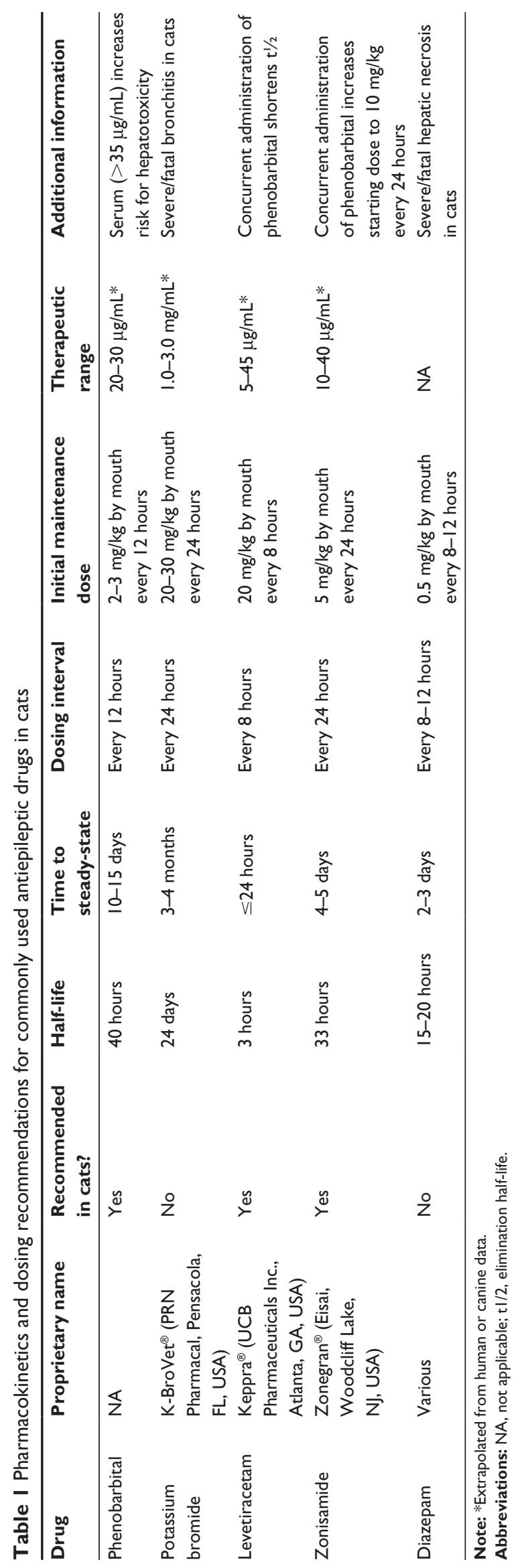


reversible with discontinuation of treatment. ${ }^{11,31}$ The protocol for therapeutic monitoring of cats receiving $\mathrm{PB}$ at the author's institution includes a baseline $\mathrm{CBC}$ and chemistry profile before initiating therapy, as well as repeating these tests in combination with a serum PB concentration 3 weeks after starting treatment. $\mathrm{CBC}$, chemistry profile, and $\mathrm{PB}$ levels are then repeated once or twice yearly. PB serum concentrations are also measured 3 weeks after any adjustment in dose.

Potassium bromide is a commonly used AED in dogs but is infrequently used in cats according to a recent survey of general veterinary practitioners. ${ }^{25}$ Potassium bromide is not recommended for use in cats because of a high incidence of adverse effects, and in particular severe allergic bronchial disease, which can be fatal. ${ }^{30,32}$

Zonisamide $(\mathrm{ZN})$ is a sulfonamide drug that exerts its anticonvulsant effects by blocking calcium channels and voltage-gated sodium channels in the brain. ${ }^{33} \mathrm{ZN}$ is available in generic form as oral capsules, making it very cost-effective as a veterinary AED. Capsule size may limit its use in cats weighing less than $5 \mathrm{~kg}$. ZN has an elimination half-life of 33 hours in cats, indicating that once-daily dosing may be appropriate. ${ }^{22,33} \mathrm{ZN}$ is metabolized primarily by the liver but appears clinically to have fewer hepatotoxic effects than $\mathrm{PB}$ and is not an inducer of CYP450. There are no reports in the veterinary literature of bone marrow or liver toxicity in cats receiving $\mathrm{ZN}$, but there are sporadic reports of adverse clinical effects in dogs. These include the development of renal tubular acidosis, acute idiosyncratic hepatic necrosis, and nonspecific drug-induced hepatopathy. ${ }^{34-36} \mathrm{ZN}$ does not cause polyuria, polydipsia, polyphagia, or sedation in most dogs and cats, making it an attractive alternative to $\mathrm{PB}$ therapy in cases in which these adverse effects are of concern. Therapeutic monitoring of ZN serum concentrations can be performed at commercial veterinary laboratories, although routine monitoring of drug concentration is not performed by most veterinary neurologists. This is because of the lack of established therapeutic range for $\mathrm{ZN}$ in dogs and cats, coupled with the generally mild adverse effects observed at higher doses. In a recent pharmacokinetic study evaluating this drug in cats, approximately half of cats receiving a dose of $20 \mathrm{mg} / \mathrm{kg} /$ day of $\mathrm{ZN}$ developed mild adverse reactions such as gastrointestinal signs or ataxia. ${ }^{33}$ The recommended starting dose of $\mathrm{ZN}$ in cats is $5-10 \mathrm{mg} / \mathrm{kg}$ once daily, with dose escalation to a maximum of $20 \mathrm{mg} / \mathrm{kg}$ daily if dictated by a lack of seizure control. A baseline $\mathrm{CBC}$ and chemistry profile should be obtained before starting $\mathrm{ZN}$ therapy and should be repeated 2-3 weeks after starting treatment. CBC and a chemistry profile are then repeated once or twice yearly in healthy patients with adequate seizure control. The efficacy of $\mathrm{ZN}$ to control feline seizures has not been evaluated but is anecdotally equivalent to other available AEDs.

Levetiracetam (LEV) is an AED with a novel mechanism of action involving inhibition of the synaptic vesicle protein SV2A to limit neuronal hyperexcitability. ${ }^{37} \mathrm{LEV}$ is primarily excreted in the urine, with limited hepatic metabolism in dogs. ${ }^{38}$ The same is assumed in cats but has not been evaluated. Limited hepatic metabolism makes LEV an attractive drug for veterinary patients with liver failure or dysfunction. In dogs and people, the pharmacokinetics of LEV are significantly altered in patients concurrently receiving PB. ${ }^{39,40}$ The pharmacokinetics of LEV has been previously evaluated in cats and indicates an elimination half-life of approximately 3 hours. ${ }^{7}$ LEV is well tolerated in cats, and the only adverse effects noted in a small clinical study were mild self-limiting inappetence and lethargy. ${ }^{7} \mathrm{LEV}$ is available in generic forms as an oral solution, oral tablet, and injectable preparation. The starting dose for LEV in cats is the same as for dogs, at $20 \mathrm{mg} / \mathrm{kg}$ orally. The drug must be administered every 8 hours because of its short elimination half-life. An extended-release form of LEV is commercially available, but tablet size and inability to split tablets preclude its use in cats. There are no published reports of significant adverse effects associated with LEV in veterinary patients; however, the author's experience is that patients with significant intracranial disease may rarely experience an unacceptable level of sedation while receiving LEV. Because of the high margin of safety, coupled with a poorly defined therapeutic range for veterinary patients, routine therapeutic drug monitoring is not performed by most clinicians. In patients with poor seizure control or patients concurrently receiving $\mathrm{PB}$, obtaining trough serum LEV levels may inform dosing recommendations.

Historically, many practitioners have used oral benzodiazepines as a maintenance AED in cats. ${ }^{25}$ This is likely related to the previous limited availability of safe, cost-effective alternatives to PB. Benzodiazepines such as diazepam exert their anticonvulsant effects via the $\mathrm{GABA}_{\mathrm{A}}$ chloride channel, with secondary effects at voltage-gated sodium channels. ${ }^{41}$ Oral benzodiazepines are ineffective as maintenance AEDs in dogs because tolerance is quickly developed. Anecdotal reduced tolerance in cats and the drug's prolonged half-life in cats compared with dogs have made diazepam an attractive second-choice AED in cats refractory to PB. Oral administration of diazepam has been associated with an idiosyncratic, potentially fatal hepatotoxicosis. ${ }^{42}$ For this reason, and because of the increased availability of other AEDs that are safe for cats, the use of oral diazepam is not recommended 
for chronic treatment of feline seizures. Intravenous benzodiazepines are still considered a first-line drug for emergency seizure management in cats, and injectable diazepam has not been reported to cause hepatic necrosis in cats. ${ }^{22}$

\section{Conclusion}

Seizures in cats may be caused by genetic, structural, or metabolic abnormalities. Primary epilepsy, although once thought nonexistent in cats, occurs at a lower frequency than what is observed in dogs. Because of this, a thorough diagnostic work-up is warranted in any cat presenting with new-onset seizures. Most commercially available AEDs, with the exception of potassium bromide and oral diazepam, are safe in cats and appear to have good efficacy. Therapeutic monitoring protocols will be dictated by the AED chosen and the health status of the patient.

\section{Disclosure}

The author reports no conflicts of interest in this work.

\section{References}

1. Schriefl S, Steinberg TA, Matiasek K, Ossig A, Fenske N, Fischer A. Etiologic classification of seizures, signalment, clinical signs, and outcome in cats with seizure disorders: 91 cases (2000-2004). $J$ Am Vet Med Assoc. 2008;233(10):1591-1597.

2. Wahle AM, Brühschwein A, Matiasek K, et al. Clinical characterization of epilepsy of unknown cause in cats. J Vet Intern Med. 2014;28(1):182-188.

3. Pákozdy A, Leschnik M, Sarchahi AA, Tichy AG, Thalhammer JG. Clinical comparison of primary versus secondary epilepsy in 125 cats. J Feline Med Surg. 2010;12(12):910-916.

4. Timmann D, Cizinauskas S, Tomek A, Doherr M, Vandevelde M, Jaggy A. Retrospective analysis of seizures associated with feline infectious peritonitis in cats. J Feline Med Surg. 2008;10(1):9-15.

5. Pakozdy A, Gruber A, Kneissl S, Leschnik M, Halasz P, Thalhammer JG. Complex partial cluster seizures in cats with orofacial involvement. J Feline Med Surg. 2011;13(10):687-693.

6. Marioni-Henry K, Monteiro R, Behr S. Complex partial orofacial seizures in English cats. Vet Rec. 2012;170(18):471.

7. Bailey KS, Dewey CW, Boothe DM, Barone G, Kortz GD. Levetiracetam as an adjunct to phenobarbital treatment in cats with suspected idiopathic epilepsy. J Am Vet Med Assoc. 2008;232(6): 867-872.

8. Mariani CL. Terminology and classification of seizures and epilepsy in veterinary patients. Top Companion Anim Med. 2013;28(2):34-41.

9. Moore SA. A clinical and diagnostic approach to the patient with seizures. Top Companion Anim Med. 2013;28(2):46-50.

10. Seppälä EH, Jokinen TS, Fukata M, et al. LGI2 truncation causes a remitting focal epilepsy in dogs. PLoS Genet. 2011;7(7):e1002194.

11. Quesnel AD, Parent JM, McDonell W, Percy D, Lumsden JH. Diagnostic evaluation of cats with seizure disorders: 30 cases (1991-1993). J Am Vet Med Assoc. 1997;210(1):65-71.

12. Barnes HL, Chrisman CL, Mariani CL, Sims M, Alleman AR. Clinical signs, underlying cause, and outcome in cats with seizures: 17 cases (1997-2002). J Am Vet Med Assoc. 2004;225(11):1723-1726.

13. Ekenstedt KJ, Oberbauer AM. Inherited epilepsy in dogs. Top Companion Anim Med. 2013;28(2):51-58.

14. Schwab S, Herden C, Seeliger F, et al. Non-suppurative meningoencephalitis of unknown origin in cats and dogs: an immunohistochemical study. J Comp Pathol. 2007;136(2-3):96-110.
15. Pakozdy A, Halasz P, Klang A, et al. Suspected limbic encephalitis and seizure in cats associated with voltage-gated potassium channel (VGKC) complex antibody. J Vet Intern Med. 2013;27(1):212-214.

16. Tomek A, Cizinauskas S, Doherr M, Gandini G, Jaggy A. Intracranial neoplasia in 61 cats: localisation, tumour types and seizure patterns. J Feline Med Surg. 2006;8(4):243-253.

17. Lipscomb VJ, Jones HJ, Brockman DJ. Complications and long-term outcomes of the ligation of congenital portosystemic shunts in 49 cats. Vet Rec. 2007;160(14):465-470.

18. Greene SN, Bright RM. Insulinoma in a cat. J Small Anim Pract. 2008;49(1):38-40.

19. Berg AT, Berkovic SF, Brodie MJ, et al. Revised terminology and concepts for organization of seizures and epilepsies: report of the ILAE Commission on Classification and Terminology, 2005-2009. Epilepsia. 2010;51(4):676-685.

20. Kuwabara T, Hasegawa D, Ogawa F, et al. A familial spontaneous epileptic feline strain: a novel model of idiopathic/genetic epilepsy. Epilepsy Res. 2010;92(1):85-88.

21. de Lahunta A, Glass E. Seizure disorders: narcolepsy. In: de Lahunta, and Glass E, editors. Veterinary Neuroanatomy and Clinical Neurology. 3rd ed. St Louis: Saunders Elsevier; 2009:454-475.

22. Smith Bailey K, Dewey CW. The seizuring cat. Diagnostic work-up and therapy. J Feline Med Surg. 2009;11(5):385-394.

23. de Lahunta A, and Glass E. The neurologic examination. In: de Lahunta, and Glass E, editors. Veterinary Neuroanatomy and Clinical Neurology. 3rd ed. Missouri: Saunders Elsevier; 2009:487-501.

24. Pozza ME, Stella JL, Chappuis-Gagnon AC, Wagner SO, Buffington CA. Pinch-induced behavioral inhibition ('clipnosis') in domestic cats. J Feline Med Surg. 2008;10(1):82-87.

25. Kluger EK, Malik R, Govendir M. Veterinarians' preferences for anticonvulsant drugs for treating seizure disorders in dogs and cats. Aust Vet J. 2009;87(11):445-449.

26. Podell M. Antiepileptic drug therapy and monitoring. Top Companion Anim Med. 2013;28(2):59-66.

27. Cochrane SM, Parent JM, Black WD, Allen DG, Lumsden JH. Pharmacokinetics of phenobarbital in the cat following multiple oral administration. Can J Vet Res. 1990;54(3):309-312.

28. Levitski RE, Trepanier LA. Effect of timing of blood collection on serum phenobarbital concentrations in dogs with epilepsy. J Am Vet Med Assoc. 2000;217(2):200-204.

29. Schwartz-Porsche D, Löscher W, Frey HH. Therapeutic efficacy of phenobarbital and primidone in canine epilepsy: a comparison. $J$ Vet Pharmacol Ther. 1985;8(2):113-119.

30. Dewey CW. Anticonvulsant therapy in dogs and cats. Vet Clin North Am Small Anim Pract. 2006;36(5):1107-1127, vii.

31. Ducote JM, Coates JR, Dewey CW, Kennis RA. Suspected hypersensitivity to phenobarbital in a cat. J Feline Med Surg. 1999;1(2):123-126.

32. Boothe DM, George KL, Couch P. Disposition and clinical use of bromide in cats. $J$ Am Vet Med Assoc. 2002;221(8):1131-1135.

33. Hasegawa D, Kobayashi M, Kuwabara T, Ohmura T, Fujita M, Orima H. Pharmacokinetics and toxicity of zonisamide in cats. $J$ Feline Med Surg. 2008;10(4):418-421.

34. Cook AK, Allen AK, Espinosa D, Barr J. Renal tubular acidosis associated with zonisamide therapy in a dog. J Vet Intern Med. 2011;25(6):1454-1457.

35. Miller ML, Center SA, Randolph JF, Lepherd ML, Cautela MA, Dewey CW. Apparent acute idiosyncratic hepatic necrosis associated with zonisamide administration in a dog. J Vet Intern Med. 2011;25(5):1156-1160.

36. Schwartz M, Muñana KR, Olby NJ. Possible drug-induced hepatopathy in a dog receiving zonisamide monotherapy for treatment of cryptogenic epilepsy. J Vet Med Sci. 2011;73(11):1505-1508.

37. Hovinga CA. Levetiracetam: a novel antiepileptic drug. Pharmacotherapy. 2001;21(11):1375-1388.

38. Moore SA, Muñana KR, Papich MG, Nettifee-Osborne J. Levetiracetam pharmacokinetics in healthy dogs following oral administration of single and multiple doses. Am J Vet Res. 2010;71(3):337-341. 
39. Moore SA, Muñana KR, Papich MG, Nettifee-Osborne JA. The pharmacokinetics of levetiracetam in healthy dogs concurrently receiving phenobarbital. J Vet Pharmacol Ther. 2011;34(1):31-34.

40. Perucca E, Gidal BE, Baltès E. Effects of antiepileptic comedication on levetiracetam pharmacokinetics: a pooled analysis of data from randomized adjunctive therapy trials. Epilepsy Res. 2003;53(1-2):47-56.
41. Calcaterra NE, Barrow JC. Classics in chemical neuroscience: diazepam (valium). ACS Chem Neurosci. 2014;5(4):253-260.

42. Center SA, Elston TH, Rowland PH, et al. Fulminant hepatic failure associated with oral administration of diazepam in 11 cats. $J$ Am Vet Med Assoc. 1996;209(3):618-625.

\section{Publish your work in this journal}

Veterinary Medicine: Research and Reports is an international, peer-reviewed, open access journal publishing original research, case reports, editorials, reviews and commentaries on all areas of veterinary medicine. The manuscript management system is completely online and includes a very quick and fair peer-review system.
Visit http://www.dovepress.com/testimonials.php to read real quotes from published authors. 